\title{
Comparison of expectations between veterinarians and veterinary nurses in tasks and responsibilities in clinical practice.
}

LC Harvey*§, and Dr. KE Cameron*

*Environmental and Animal Sciences, Unitec Institute of Technology, 139 Carrington Road, Mt Albert Auckland, New Zealand §Author for correspondence. Email: Iharvey@unitec.ac.nz 


\section{Acknowledgements}

- This research was approved by the Unitec Research Ethics Committee, Auckland, New Zealand (UREC Registration Number: 2014-1070).

- We would like to thank the NZVA and NZVNA for distributing the survey and the Unitec Research and Enterprise office for assisting with funding this project. 


\section{The study}

To investigate the differences in perspectives between Veterinary Nurses (VNs) and Veterinarians in clinical practice in New Zealand.

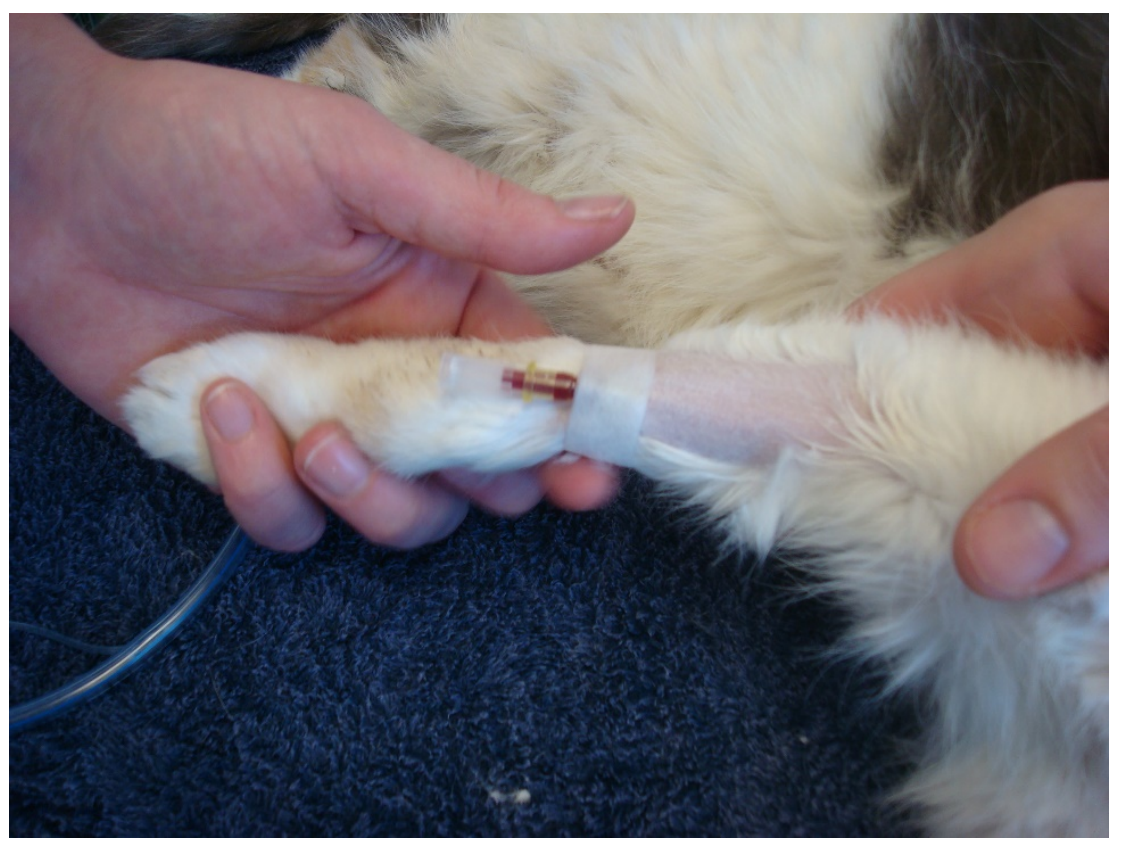




\section{Why?}

- Is this situation familiar? A vet doing a task you know you are capable of, and maybe even better at?

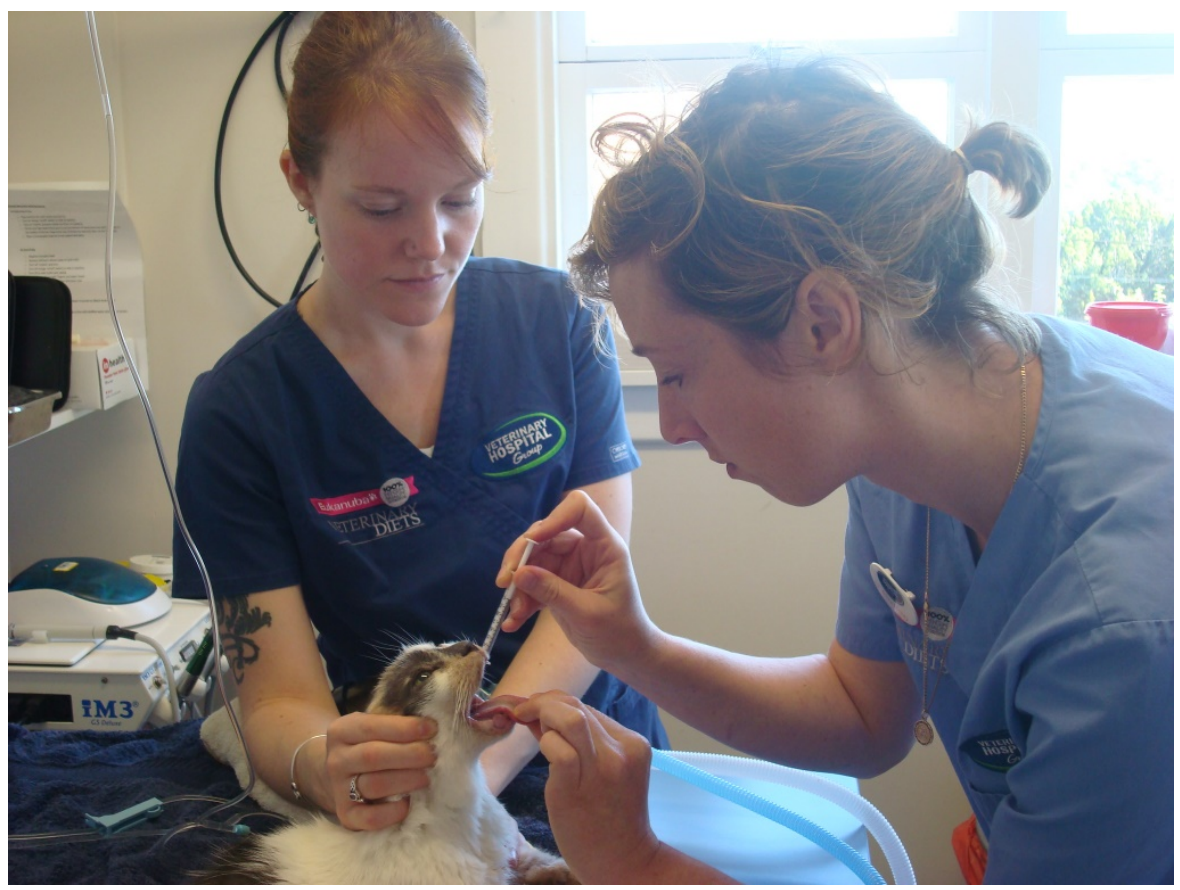





\section{The tasks - VN tasks}

- Taking a blood sample

- Placement of IV catheters

- Set-up of IV fluids

- Vet Nurse consults

- Clinical exams

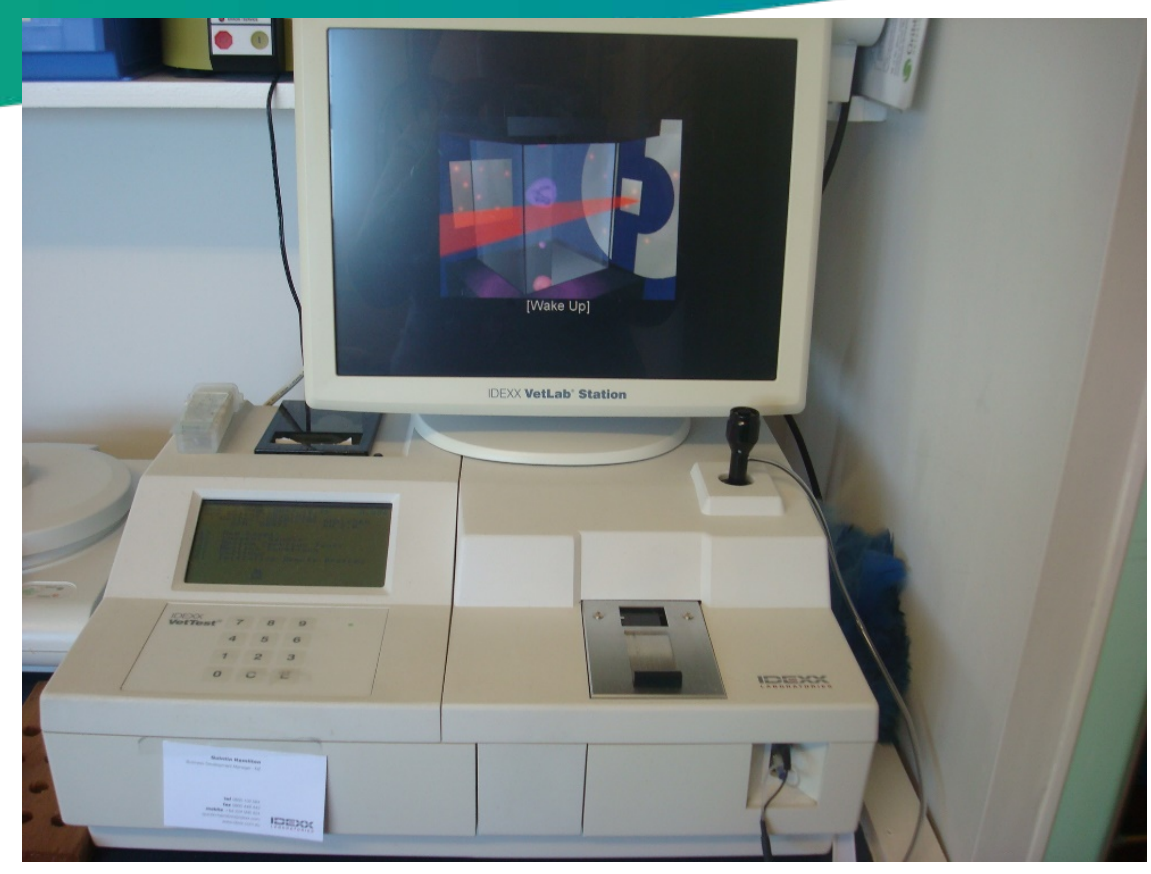

- Diagnostic testing using Idexx/Vetscan or similar 


\section{The tasks - VN tasks continued}

- Urinalysis

- Microscopy

- Taking radiographs

- Dental scale and polish

- Assisting with surgery

- Monitoring anaesthetics

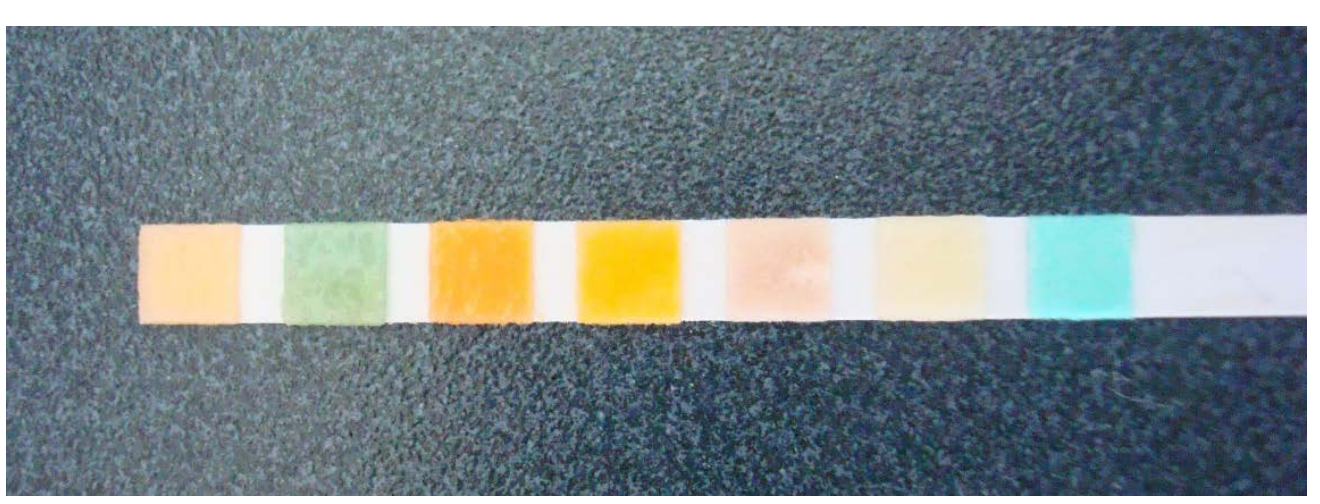




\section{The tasks - Vet tasks}

- Cat neuters

- Dental extractions 


\section{The tasks - why}

- VN tasks were chosen based on those currently taught as part of the diploma level qualification in New Zealand, as well as those often undertaken by VNs known to the researchers. 


\section{Why?}

- VNs are trained and assessed for competency in a range of clinical tasks

- VNs have reported that Vets are completing many of these clinical tasks instead of veterinary nurses

- VNs may not be being utilised to their full capacity 


\section{Historical observations}

- In 1998, Janet Molyneux listed the skills VNs in practice should be undertaking.

- In 2010, at the 3rd AVA/NZVA Pan Pacific Veterinary Conference, Veterinarian Dr Karen Koks talked about the under-utilisation of VNs in practice, and how to make better use of VNs.

- In 2012 at the New Zealand Veterinary Nursing Association annual conference, Veterinarian Dr Linda Sorensen, made a point of saying that "the goal of the nurse-centred clinic is to fully utilise the nursing staff within legal limits". 


\section{How?}

- Web-based questionnaire (SurveyMonkey)

- Anonymous

- 288 responses (March 2015)

- Response rate: $19 \%$ of the NZ veterinary nursing population $(n=288 / 1479)$

- This sample size gives a margin of error of $5 \%$, with a confidence level of $95 \%$

- Distribution

- NZVNA via email

- NZVA e-newsletter

- Social Media (Facebook) 


\section{How?}

- 38 multi-choice questions

- The questionnaire was divided into four main sections

- Demographic information

- Tasks

- Wellness

- Registration

- Questions were predominantly multi-choice with some allowing for comments. 


\section{Age}

\section{Most respondents were 20-31 years of age.}

up to 16 years

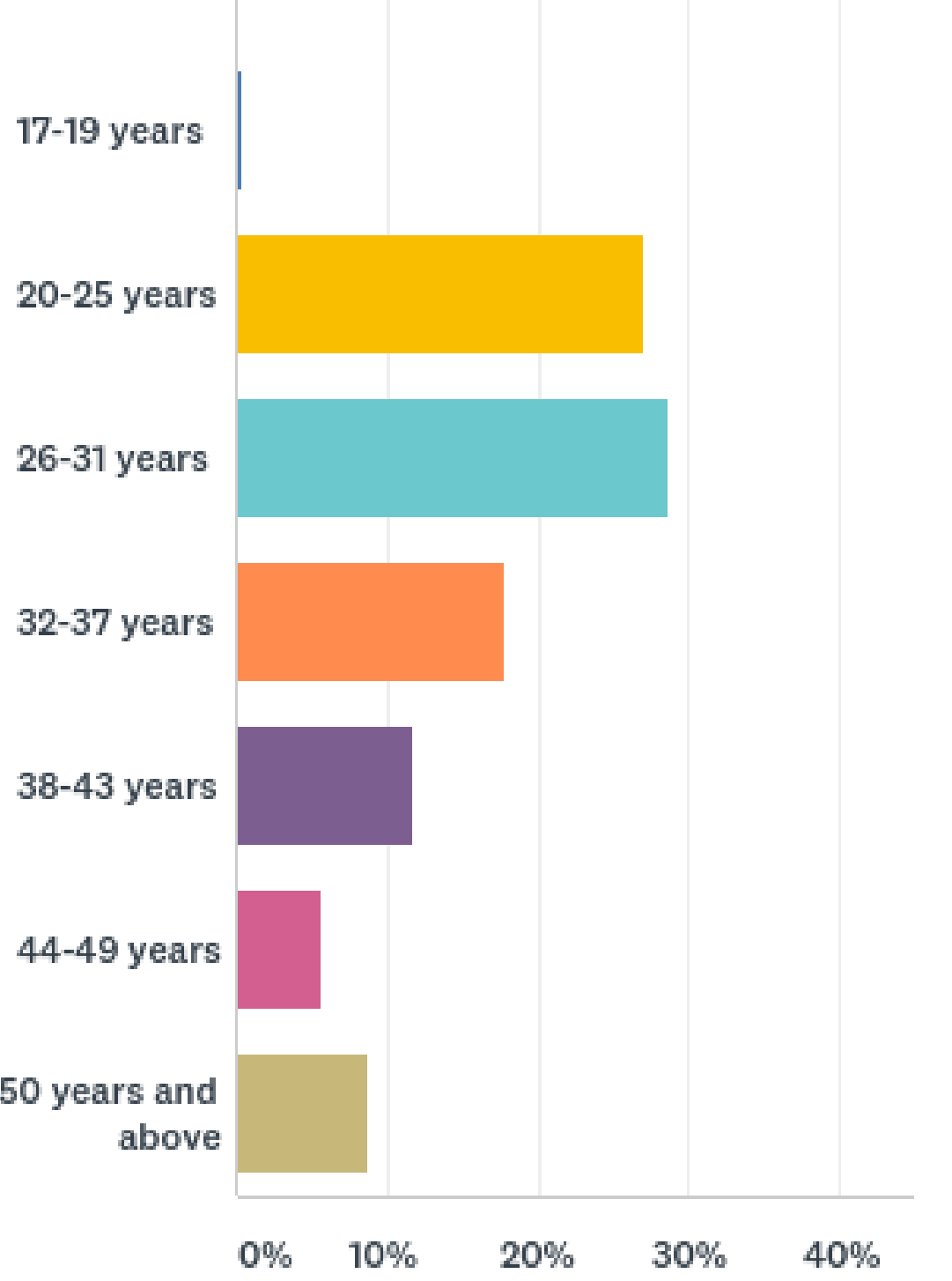




\section{Gender}

Most respondents were female

\section{Female}

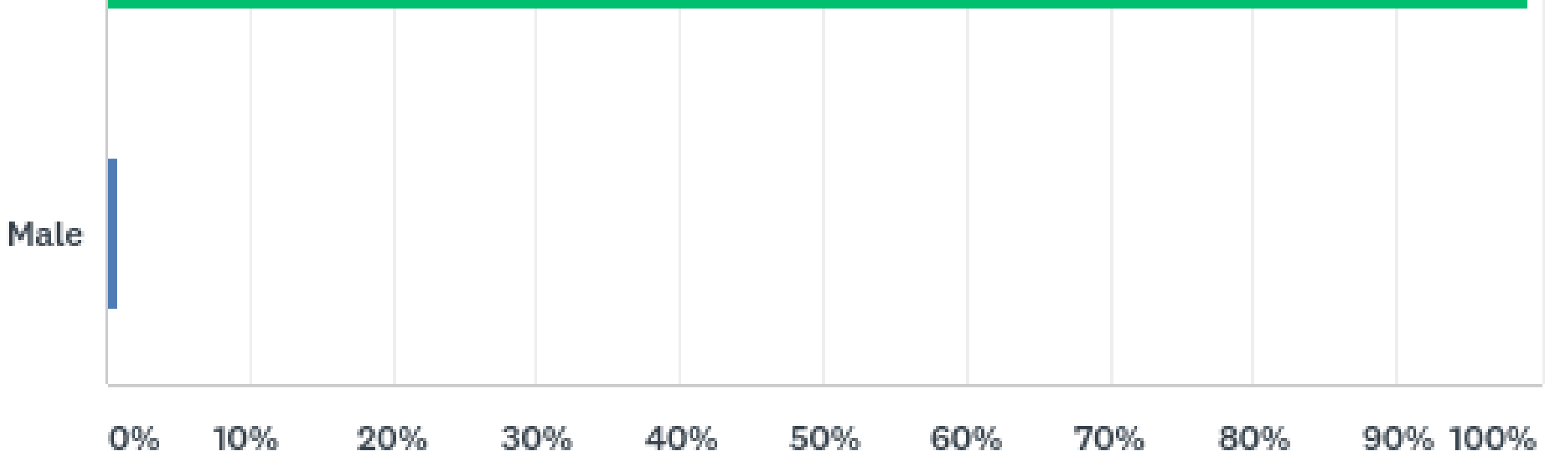




\section{Qualification}

Most respondents had a diploma level veterinary... qualification, with the majority of the remaining respondents having a certificate level qualification.

\section{Degree in Veterinary...}

\section{Diploma in} Veterinary...

Certificate in Veterinary...

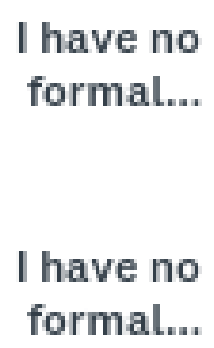




\section{Demographics summary}

- The demographics of survey respondents fits with what is observed within the profession in New Zealand 


\section{Undertaking of tasks in clinical practice, as reported by VNs}

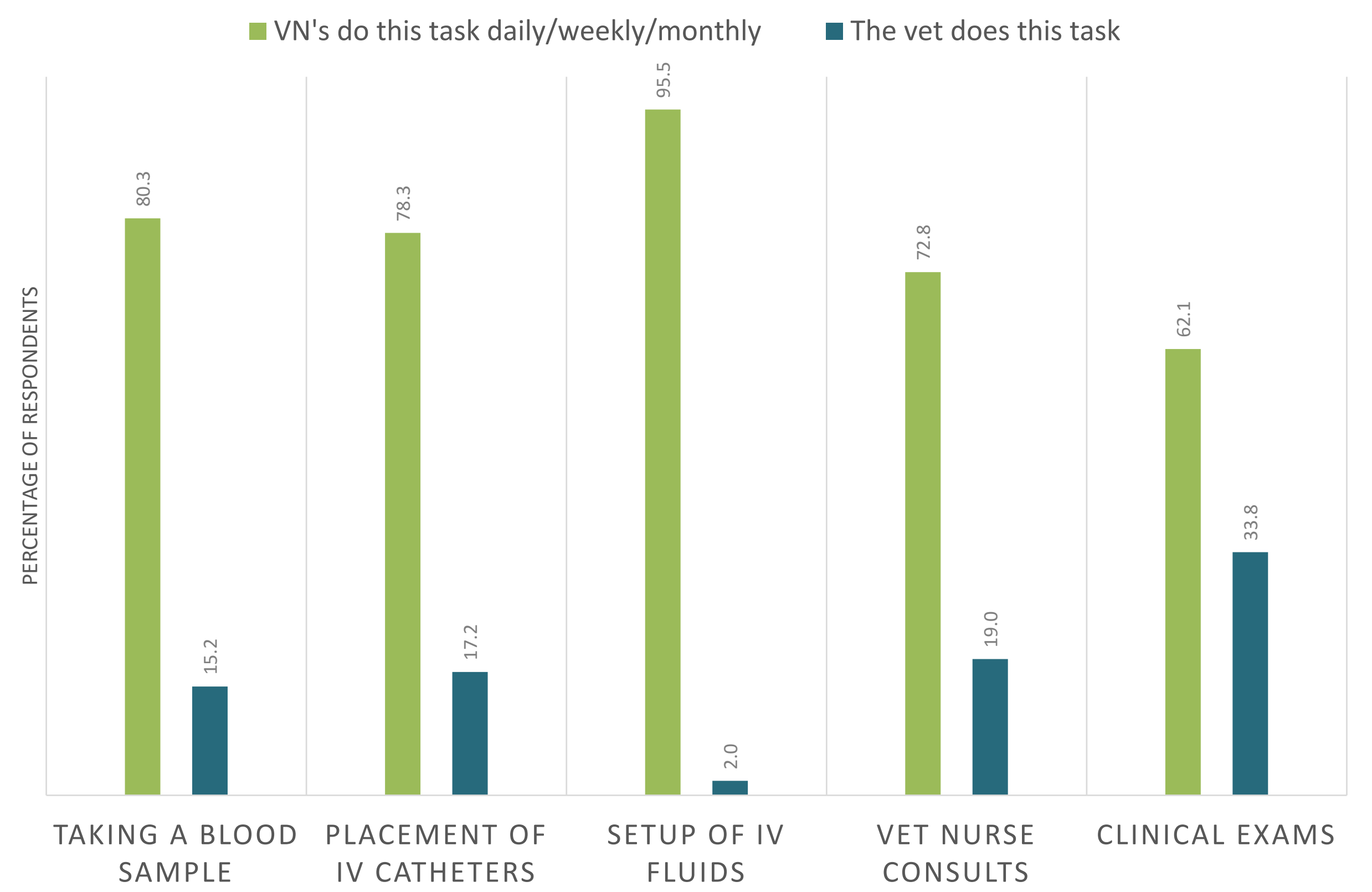




\section{Undertaking of tasks in clinical practice, as reported by VNs}

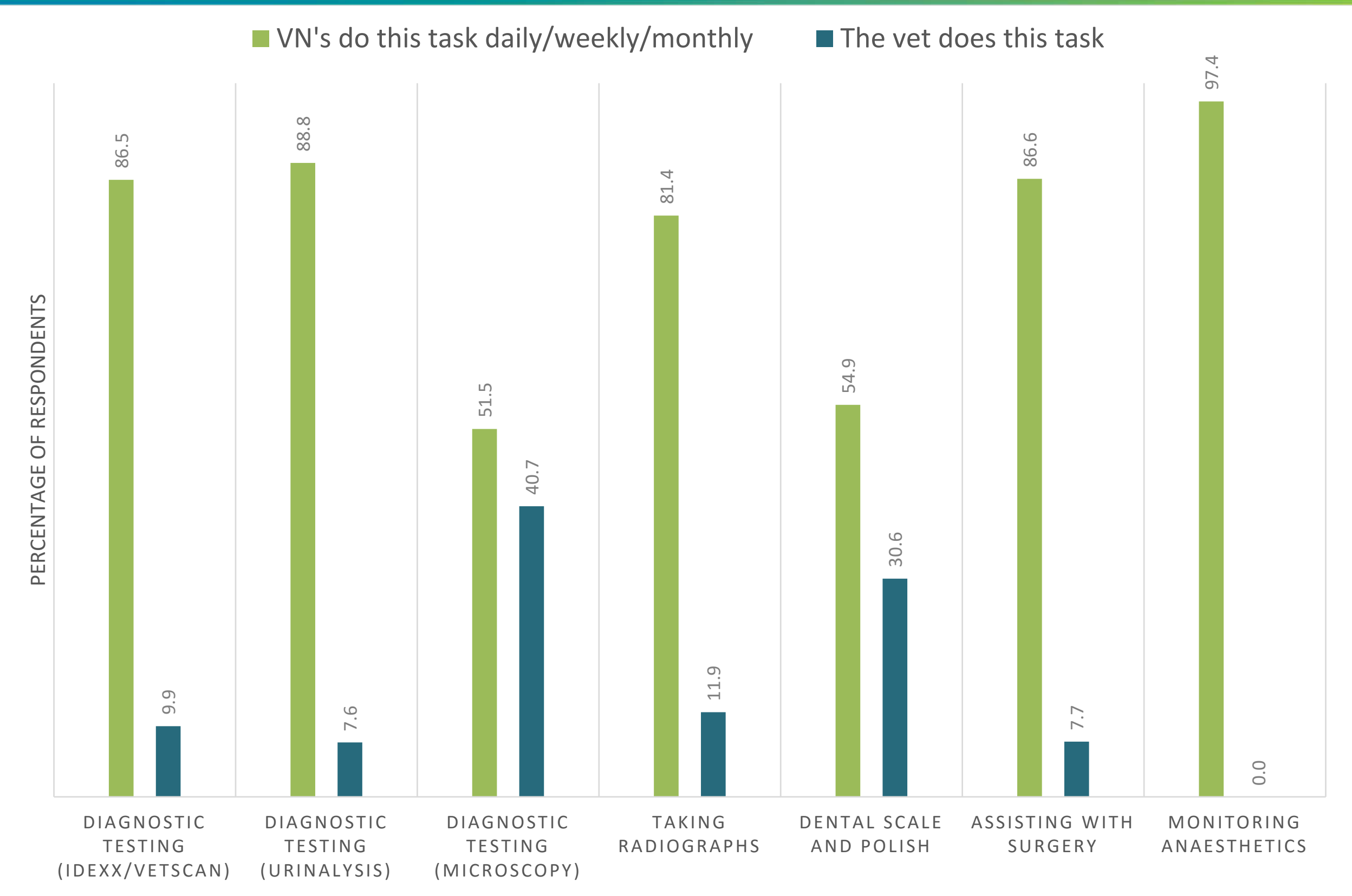




\section{Undertaking of tasks in clinical practice, as reported by VNs}

- VN's do this task daily/weekly/monthly

- The vet does this task

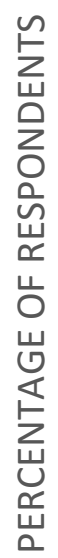

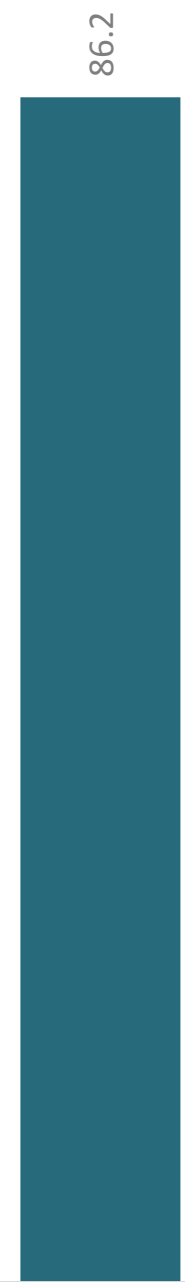




\section{What this means}

- As we would hope for, many tasks show a high level of VN involvement and low level of Vet involvement, but there are still a number of tasks with a high level of Vet input

- The results are similar (although not exactly the same) when we look at the Veterinarian responses to the same tasks. 


\section{Undertaking of tasks in clinical practice, as reported by Veterinarians}

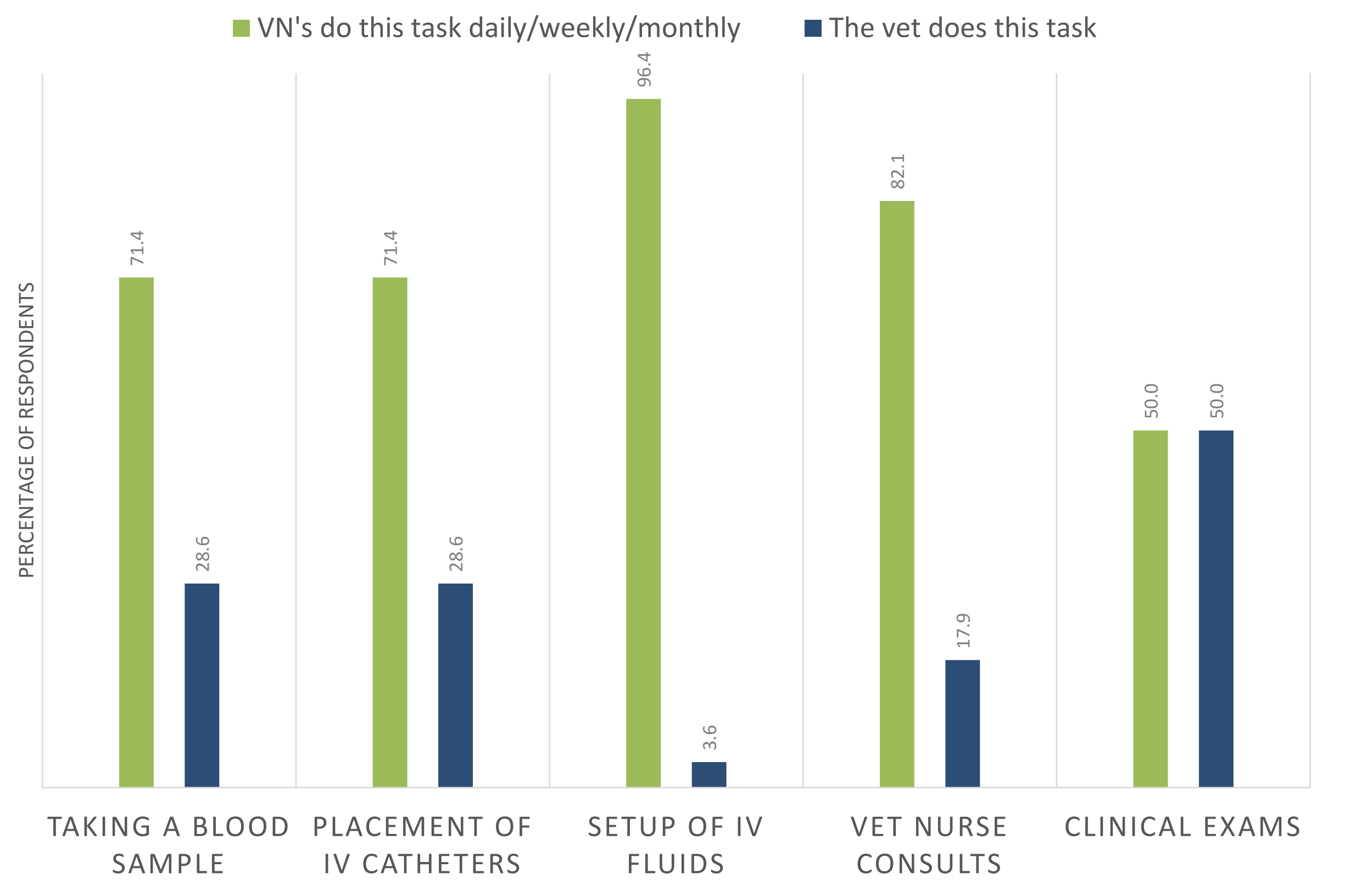




\section{Undertaking of tasks in clinical practice, as reported by Veterinarians}

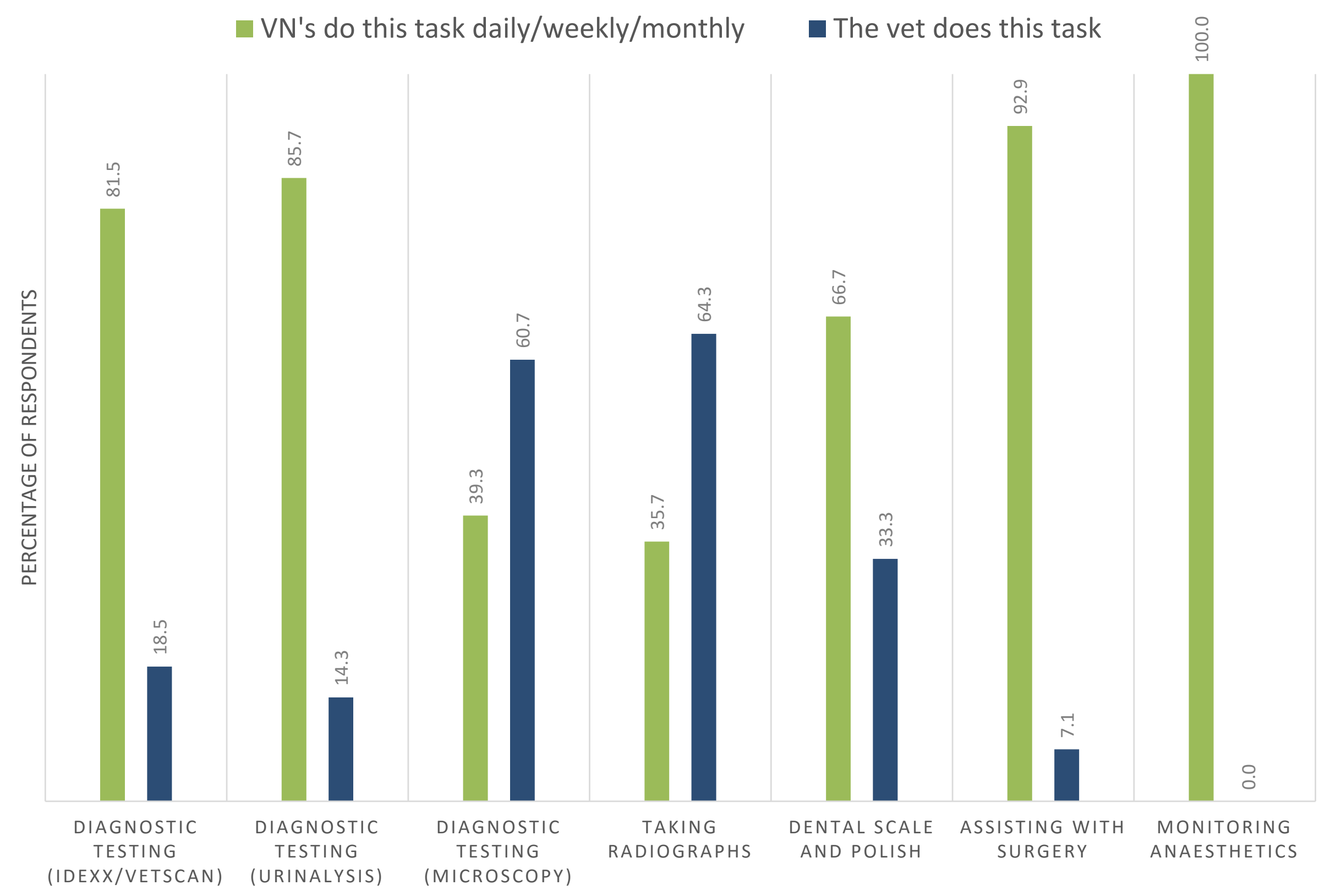




\section{Undertaking of tasks in clinical practice, as reported by Veterinarians}

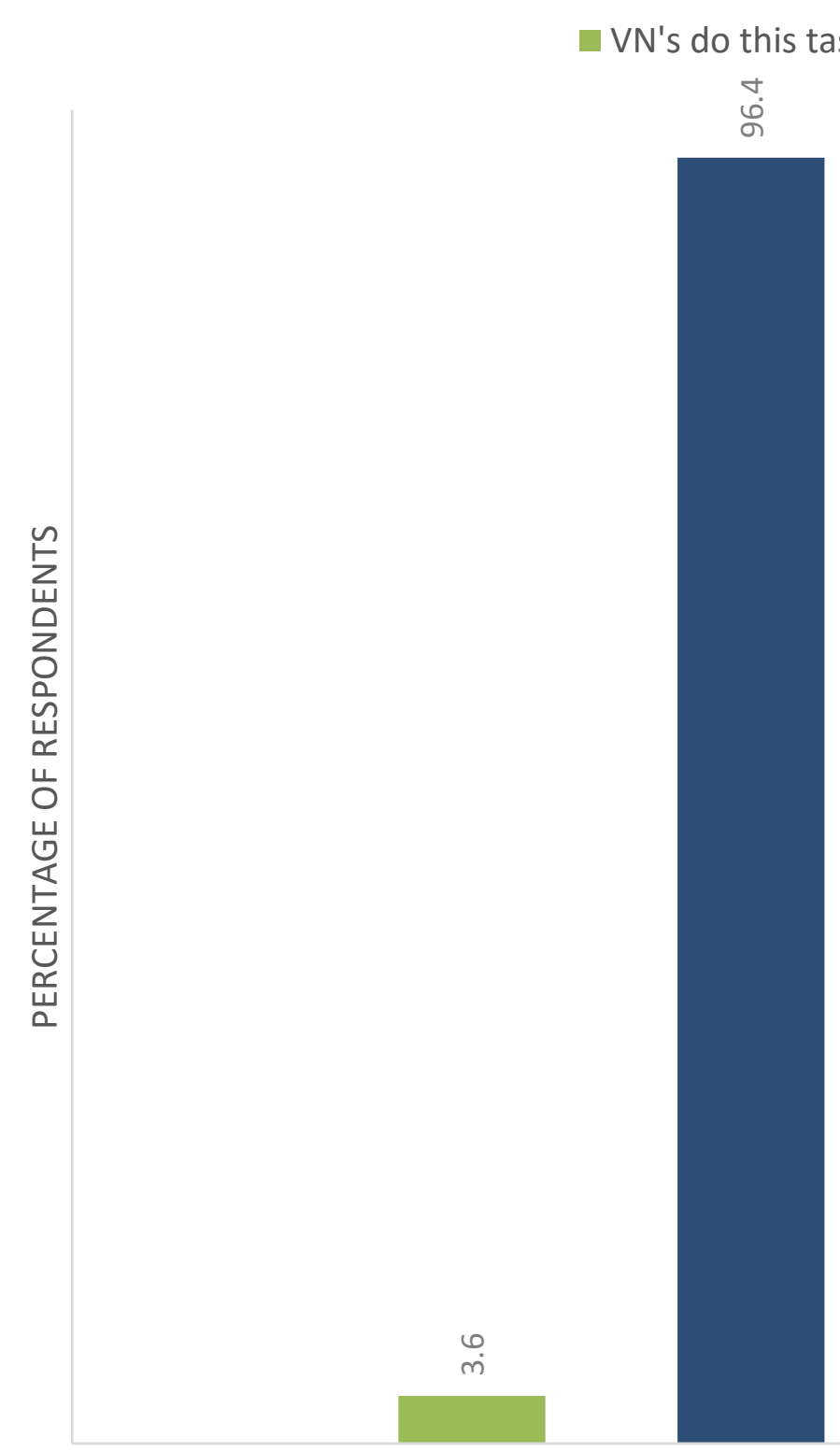

CAT NEUTERS
- The vet does this task

ஸ̊.

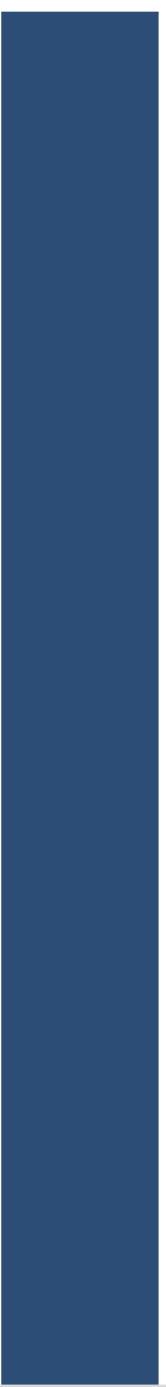

DENTAL EXTRACTIONS 


\section{Side by side comparison}

-When we look at a comparison of what VNs are doing in clinic, and what Vets want them to be doing, we see some gaps. 


\section{Comparison of task responsibility}

- VN's currently do this task (as reported by VN's)

- VN's should be undertaking this task (as reported by vets)

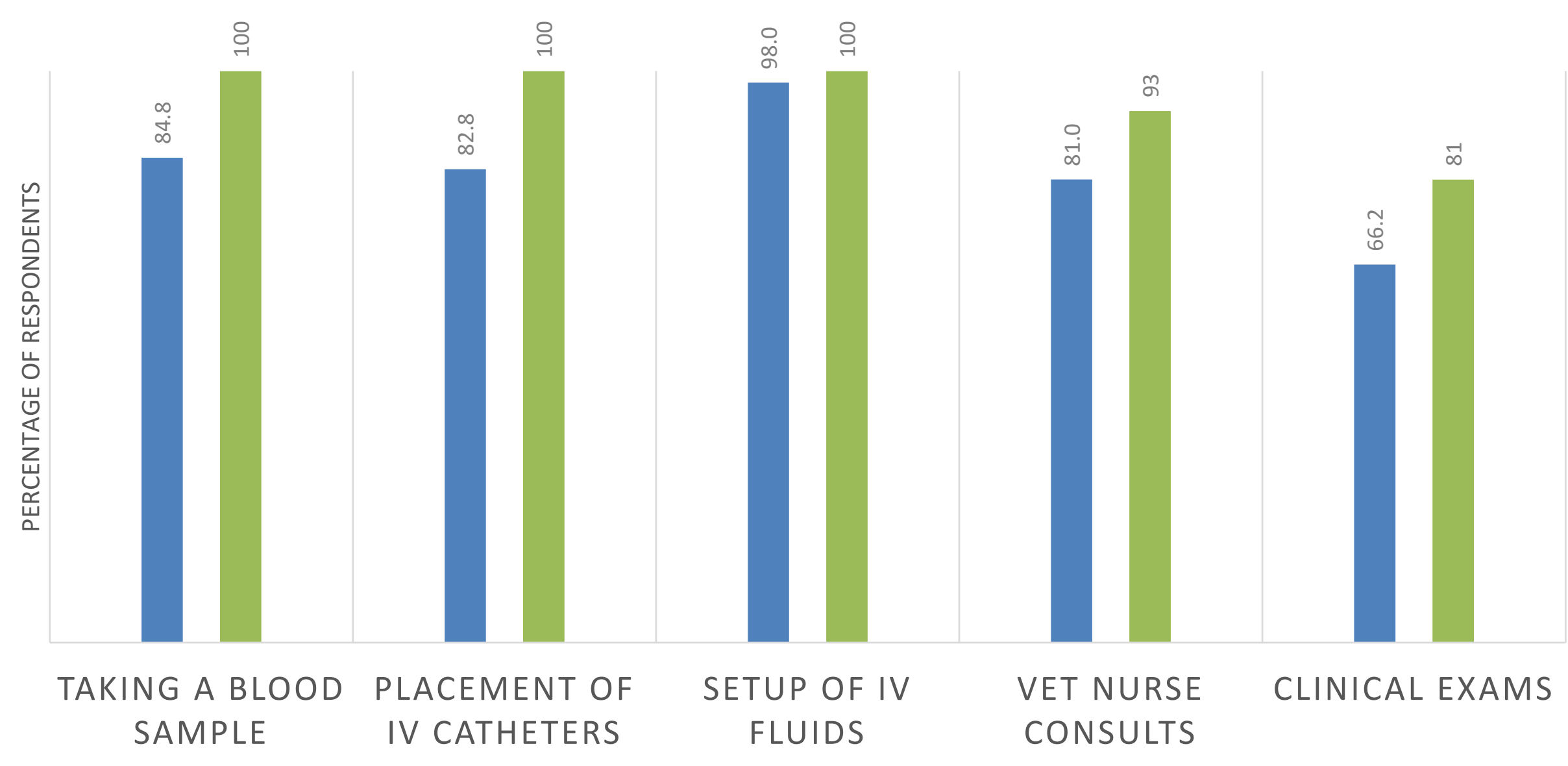




\section{Comparison of task responsibility}

- VN's currently do this task (as reported by VN's)

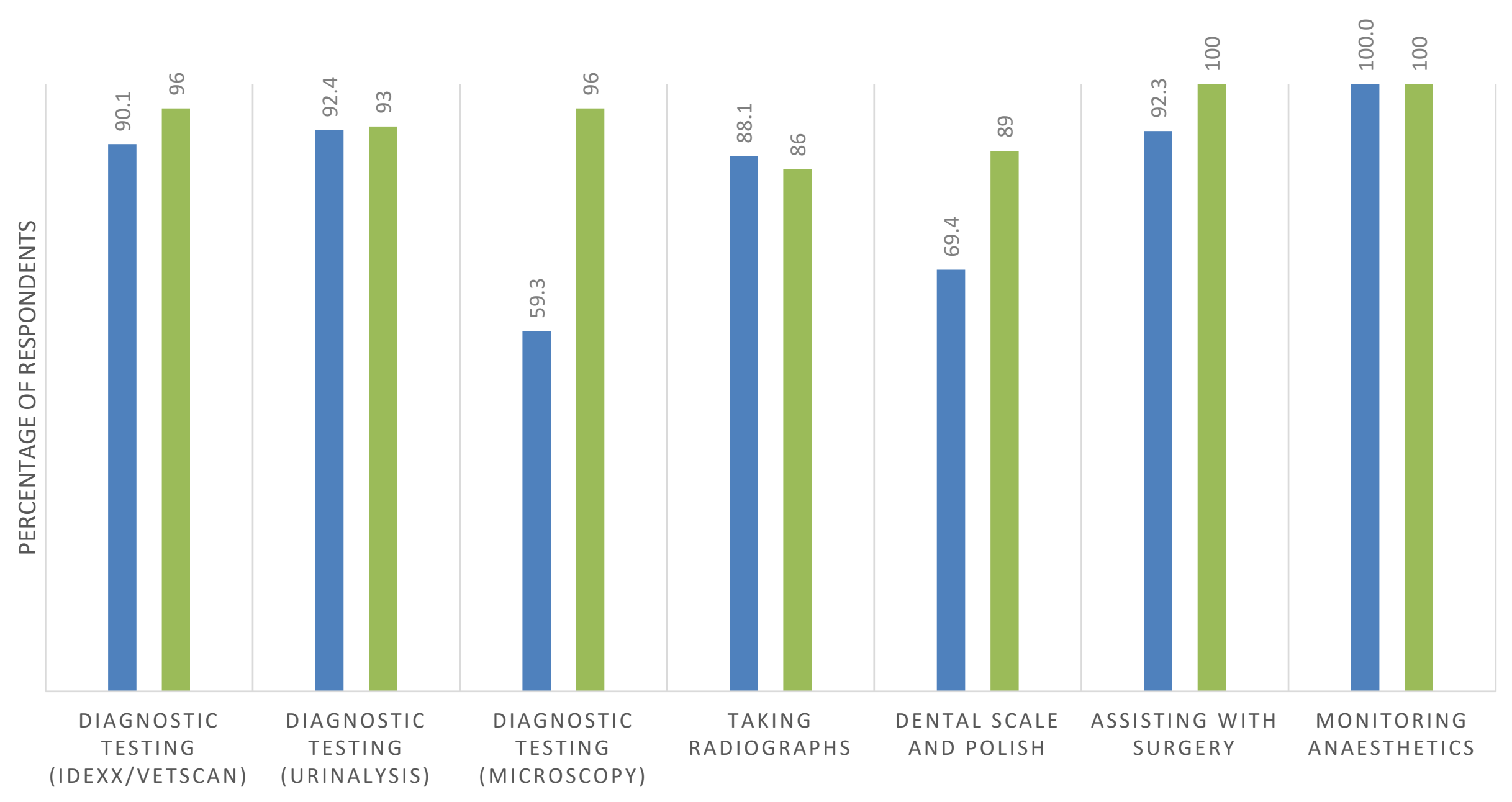




\section{Comparison of task responsibility}

- VN's currently do this task (as reported by VN's)

- VN's should be undertaking this task (as reported by vets)

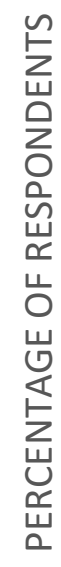

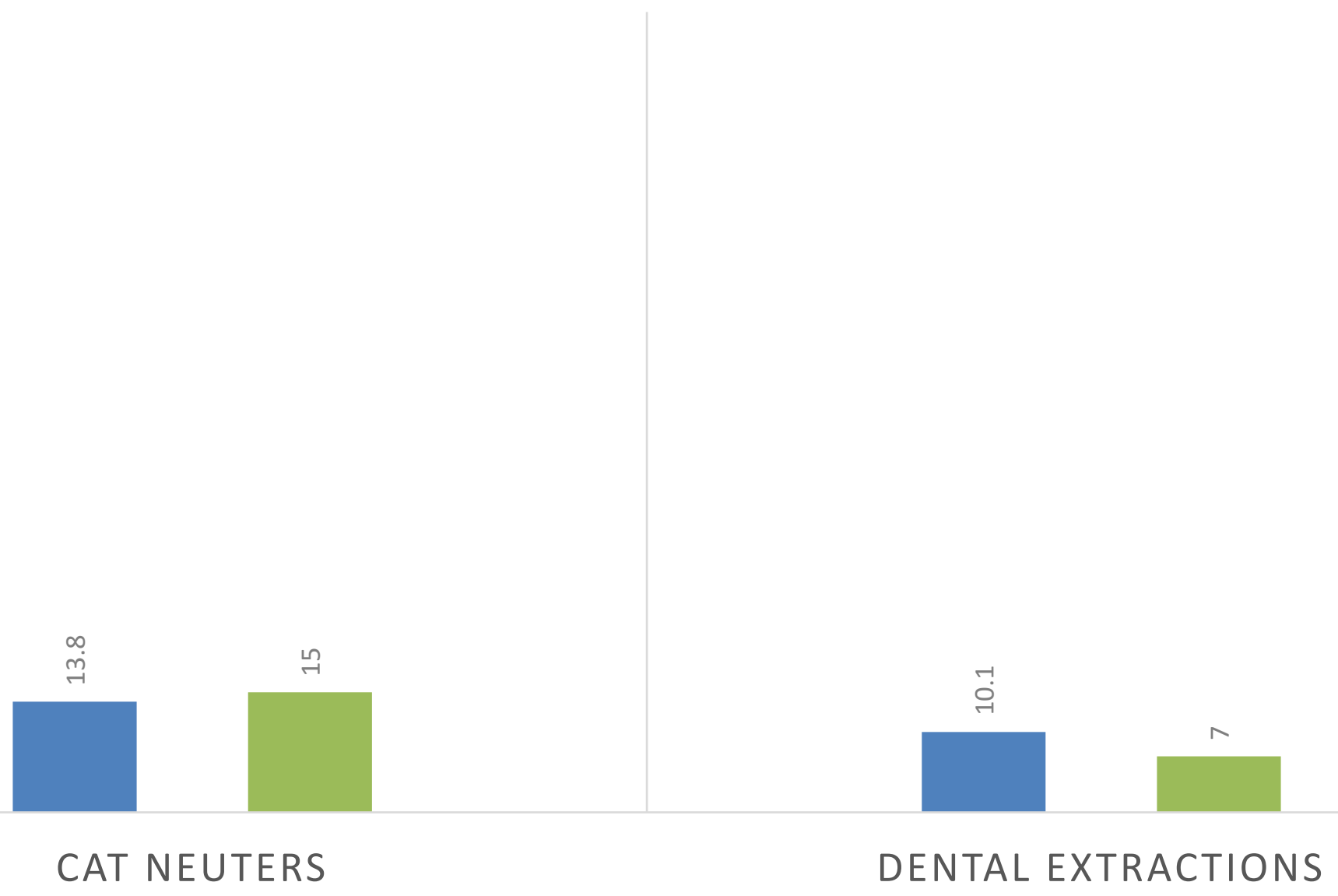




\section{What it all means}

- The results of the study show that VNs are wanting to undertake a range of clinical tasks in practice, and they have the support of Vets to do this, however the numbers of individual VNs actually completing some of these tasks is low. 


\section{Discussion}

- What we have:

- Capability

- Willingness

- What we know:

- Veterinarians want VNs to complete more tasks in clinical practice

- No obvious reason for the current disparity

- What it can mean:

- Improved wellbeing

- Financial: increase profits 


\section{Discussion}

Current guidelines regarding the limit to the tasks of a Veterinary Nurse in clinical practice is dictated by the New Zealand Animal Welfare Act 1999 ("Animal Welfare Act," 1999), and the impending amendment ("Animal Welfare Amendment Act (No 2)," 2015).

This limits 'significant surgical procedures' to Veterinarians and Veterinary students, however, the 12 Veterinary Nursing tasks discussed are permitted through current legislation. 


\section{Discussion}

Of interest (and potential concern) is the incidence of VNs reporting that they performed, but more likely, assisted, with surgical procedures such as cat neuters and dental extractions in contradiction to the Animal Welfare Act.

Regardless, clarity within the Acts and in the survey, might clear up or expose inconsistencies in the role of the VN for surgical procedures. 


\section{Discussion}

For example, subgingival scaling is a key component of dental prophylaxis yet the new amendment has classified any procedure 'below the gingival margin' such as the VN task of 'scale and polish' as being a surgical procedure only to be undertaken by a Veterinarian or Veterinary student.

This seems a backwards step in terms of advancing the VN profession. The Dental Council of New Zealand Scope of Practice for dental hygienists includes subgingival scaling, so we are not keeping up with our human healthcare counterparts. 


\section{Discussion - NZVNA Registration}

- Our study showed almost $60 \%$ support for statutory registrations of VNs

- In 2018, 31\% (435) of VNs (including LVN/VNA) were registered/listed

- Veterinarians have had mandatory registration since 1926

- Does this lack of statutory registration have anything to do with the lack of VNs doing some of these tasks?

- Will this change with compulsory registration?

- Will Vets then be more willing to give up control and responsibility to VNs? 


\section{Conclusions}

VNs are trained in tasks that they are not performing in clinical practice.

The justification for this discrepancy is the subject of a future study with the aim of designing practical support for veterinary clinics to utilize the skills of VNs. 


\section{What next?}

It would be interesting to clarify and redistribute the survey to VNs and Vets in the future to investigate whether Veterinary Nurses' 'lack of accountability' to a governing board is a reason for Veterinarians performing VN tasks. 


\section{Interesting comments from vet nurses}

- We only have one vet one nurse, although I am very capable and would ideally like to do it all of the time

- Our vets are old school and are used to doing everything themselves

- Because the vets want to do it

- The vets in my clinic think that it is their job and nurses should not be doing it

- Vets in our practice prefer to do it themselves or don't have the time to train nurses to do it 


\section{Interesting comments from vets}

- Although I would like to see the nurses expand into some of these tasks, senior vets feel they cannot and will not let them expand their role

- Our VN's do not like to take blood samples nor set up IVFT

- Nurses are not suitably trained to do these tasks

- We have final year vet students who do these 